\title{
Comparative studies on field emission properties of carbon-based materials
}

\author{
K.H. Chen ${ }^{\text {a, } *}$, J.-J. Wu ${ }^{\text {a }}$, L.C. Chen ${ }^{\text {b }}$, C.Y. Wen ${ }^{\text {b }}$, P.D. Kichambare ${ }^{\text {b }}$, F.G. Tarntair ${ }^{\text {c }}$, \\ P.F. Kuo ${ }^{\mathrm{d}}$, S.W. Chang ${ }^{\mathrm{d}}$, Y.F. Chen ${ }^{\mathrm{d}}$ \\ ${ }^{a}$ Institute of Atomic and Molecular Sciences, Academia Sinica, Taipei, Taiwan \\ ${ }^{\mathrm{b}}$ Center for Condensed Matter Sciences, National Taiwan University, Taipei, Taiwan \\ ${ }^{\mathrm{c}}$ Department of Electronic Engineering, National Chiao Tung University, Hsinchu, Taiwan \\ ${ }^{\mathrm{d}}$ Department of Physics, National Taiwan University, Taipei, Taiwan
}

\begin{abstract}
Comparative studies on field emission properties of various carbon-related materials including diamond, amorphous carbon, SiCN films, SiCN nanorods, and carbon nanotubes are presented. While diamond is well known for its potential use in cold cathodes, the emission current of a hydrogen-treated diamond film is relatively small compared to that of amorphous carbon or diamond-like-carbon film. Meanwhile, carbon nanotubes have demonstrated large emission currents at much lower threshold voltages, showing their potential for applications. However, the emission from carbon nanotubes is subjected to significant decay under long-term operation. The emission current and long-term stability can be improved using a new SiCN compound with nanorod morphology. The effective barrier height and the field enhancement factor derived from the Fowler-Nordheim equation are discussed in this paper. (C) 2000 Elsevier Science S.A. All rights reserved.
\end{abstract}

Keywords: Amorphous carbon; Carbon-based materials; Carbon nanotubes; Diamond; Field emission properties; $\mathrm{SiCN}$ films; SiCN nanorods

\section{Introduction}

In the past few years, considerable interest has been focused on the electron field emission from diamond and related materials [1-3]. Due to its negative electron affinity property, diamond has been widely recognized as the dream material for various applications from cold cathodes, flat panel displays, to a new generation of microwave tubes. Experimental data, however, indicate that non-diamond phases such as diamond-like carbon (DLC), amorphous carbon (a-C), and other nanostructural carbon demonstrate a much better field-emission property than that of diamond [4,5]. The addition of dopants into the material can substantially reduce the onset field from $30 \mathrm{~V} / \mu \mathrm{m}$ typically to a few $\mathrm{V} / \mu \mathrm{m}$, and can enhance the emission current up to hundreds of $\mu \mathrm{A} / \mathrm{cm}[2,6]$. It has been demonstrated that the introduction of an $\mathrm{sp}^{2}$ phase in the film further reduces the onset field intensity and enhances the emission current [7].

\footnotetext{
* Corresponding author. Fax: +886-2-2362-0200.

E-mail address: chenkh@po.iams.sinica.edu.tw (K.H. Chen)
}

With the advent of nanometer materials such as carbon nanotubes, the carbon-based electron field emitter experienced another rush, bringing the idea of cold-cathode closer to reality.

In this report, a comparison of materials produced in our laboratory ranging from bias-assisted carburized (BAC) silicon tips [8], SiCN nano-crystallite [9,10], $\mathrm{SiCN}$ nanorods [11], and carbon nanotubes [12] is made to elucidate the mechanisms of field emission. The correlations of the material, the field enhancement factor (geometry of the sample) and the effective barrier height (work function) to the emission current are investigated.

\section{Experimental}

\subsection{Sample preparation}

A silicon microtip array $(50 \times 50$ square and $1 \mu \mathrm{m}$ in width) of each tip was produced by standard photolithography and reactive ion etching processes. The ultra-high aspect ratio cone-shaped microtip array was 


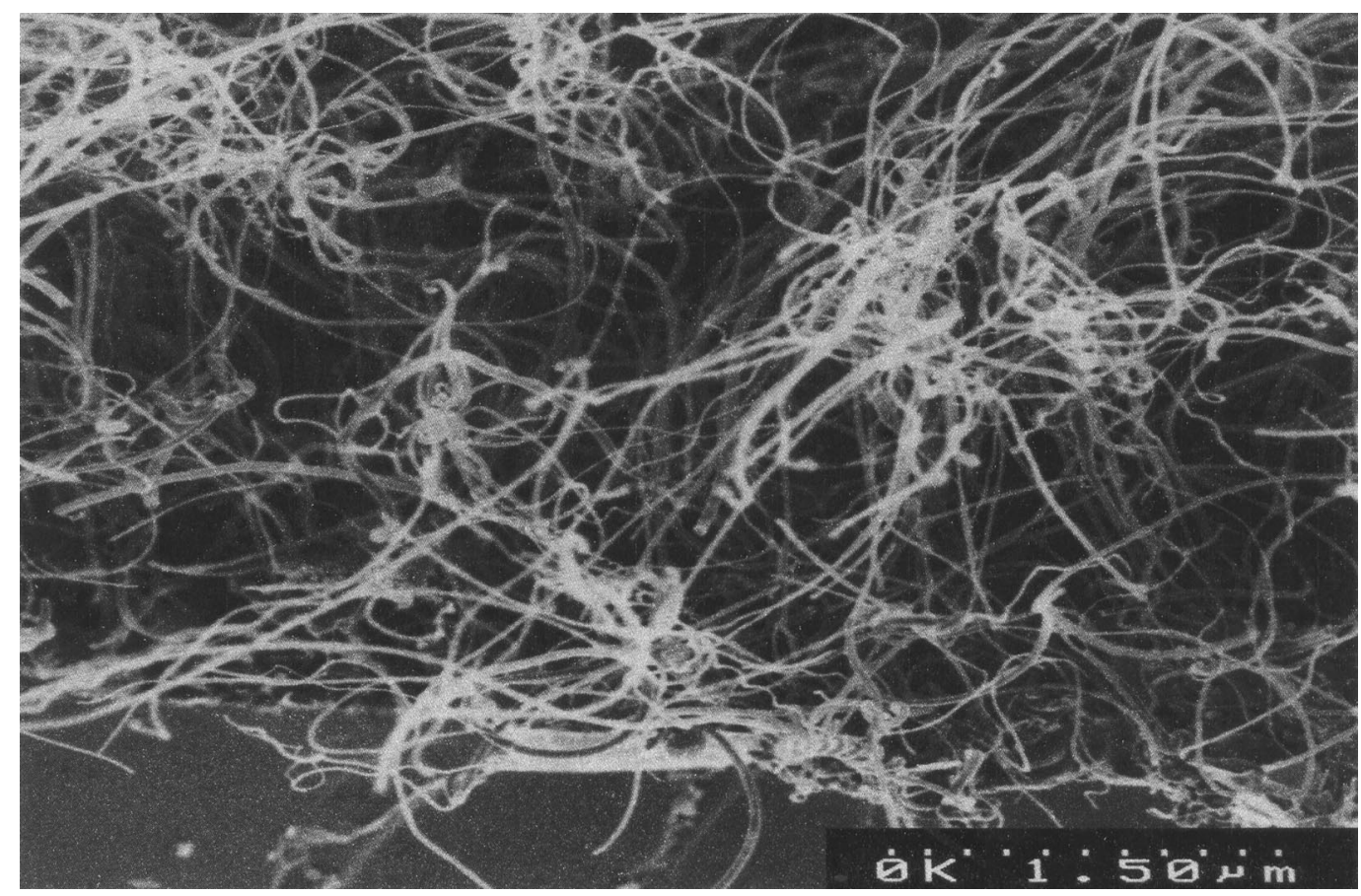

Fig. 1. Typical SEM micrograph of carbon nanotubes.

further coated with a carbon layer using the BAC process. Selective deposition of the carbon layer on the surface of sharp silicon tips was achieved by careful adjustment of the bias voltage and bias time. The optimal conditions for the BAC were $-200 \mathrm{~V}$ bias voltage for $40 \mathrm{~min}$ with $25 \% \mathrm{CH}_{4}$ in $\mathrm{H}_{2}$ using a microwave plasma CVD reactor at a substrate temperature of $650^{\circ} \mathrm{C}$. Typically, sharp tips of $50 \mathrm{~nm}$ radius were produced for the emission study.

The carbon nanotubes in this study were deposited using microwave plasma enhanced CVD operated at $1.5 \mathrm{~kW}$. An n-type Si substrate was first deposited with $300 \AA \mathrm{Fe}$ and then nanotube growth was continued in the CVD reactor with $20 \mathrm{sccm} \mathrm{CH}_{4}$ and $80 \mathrm{sccm} \mathrm{H}_{2}$ at a pressure of 50 Torr and a substrate temperature of $900^{\circ} \mathrm{C}$. The $\mathrm{Fe}$ catalytic layer is patterned to form a $50 \times 50$ array of $8 \mu \mathrm{m}$ squares with $16 \mu \mathrm{m}$ spacing. After 30 min of deposition, we observed multi-wall tubes of $10 \mu \mathrm{m}$ in length and $20-50 \mathrm{~nm}$ in diameter with a random orientation (Fig. 1).

The growth of $\mathrm{SiCN}$ films has been described in detail elsewhere [13]. In short, an electron cyclotron resonance (ECR) plasma CVD reactor operating at a microwave power of $1200 \mathrm{~W}$ with a gas ratio of $\mathrm{CH}_{3} \mathrm{NH}_{2}: \mathrm{SiH}_{4}: \mathrm{N}_{2}: \mathrm{H}_{2}=50: 1: 125: 125$ and a silicon substrate temperature of $700^{\circ} \mathrm{C}$ was utilized to deposit nano-crystalline $\mathrm{SiCN}$ films of $300 \mathrm{~nm}$ thick on a silicon substrate. The typical stoichiometry of the SiCN films

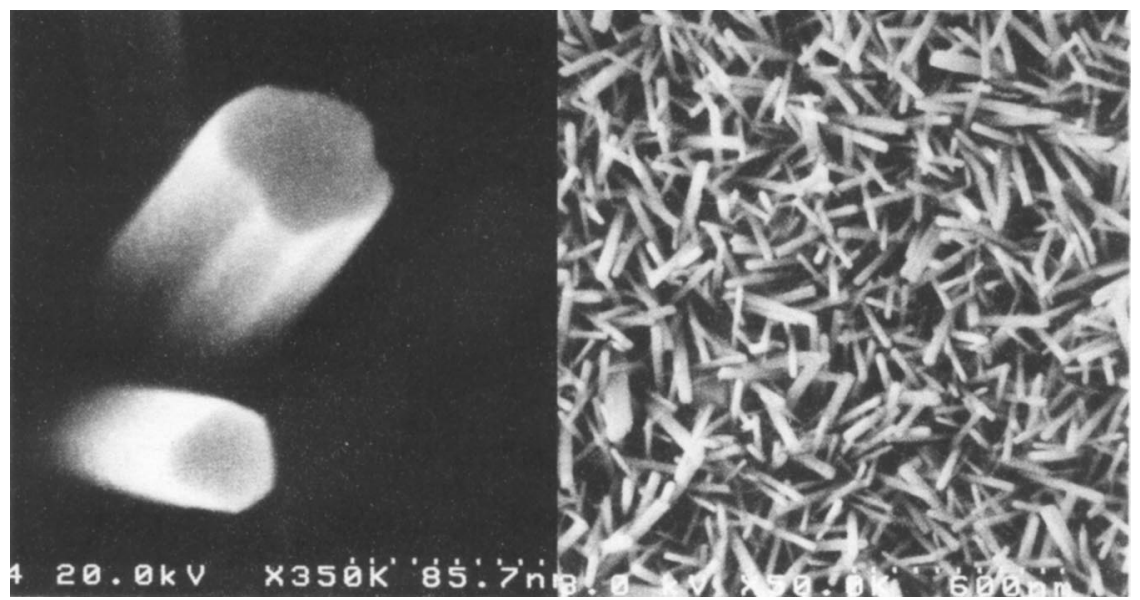

Fig. 2. Typical high-resolution SEM micrograph of SiCN nanorods. 
thus deposited was $(\mathrm{Si} ; \mathrm{C})_{3} \mathrm{~N}_{4}$ with $\mathrm{Si} / \mathrm{C}$ ranging from 1 to 2 . The films thus deposited had a hexagonal structure with typical lattice constants of $a=7.70 \AA$, and $c=$ $5.78 \AA$.

In addition to the crystalline films described above, SiCN exhibited another phase of reduced dimensionality. By using a proprietary buffer layer of $30 \mathrm{~nm}$ before regular MWPE-CVD, one-dimensional $\mathrm{SiCN}$ rods of 20-60 $\mathrm{nm}$ with an aspect ratio of 50 were deposited. A high-resolution SEM image of the SiCN nanorods is shown in Fig. 2, indicating well-faceted nanorods with a six-sided cross-section. The detailed growth mechanism and characteristics of the $\mathrm{SiCN}$ nanorods are not included in this paper and will be reported elsewhere [11].

\subsection{Field emission measurement}

The field emission characteristics were obtained using standard $I-V$ measurement under a high vacuum with a base pressure of $2 \times 10^{-7}$ Torr, as given in Fig. 3. A rectangular graphite electrode of $2 \times 3 \mathrm{~cm}^{2}$ was placed $30 \mu \mathrm{m}$ above the sample. A Keithely multimeter was employed for sourcing the voltage and measuring the current. Precautions were taken to clean the sample before measurement in order to eliminate any dust particles on the surface that might have caused false signals. During the measurement, the voltage increase was adjusted to allow a detailed study of the $I-V$ characteristics. The option to hold the voltage and record the emission current at a constant voltage makes possible, emission stability measurement, which is important for practical applications.

\subsection{Other characterization}

The morphology of the films was analyzed using a Hitachi scanning electron microscope (S-5000), which

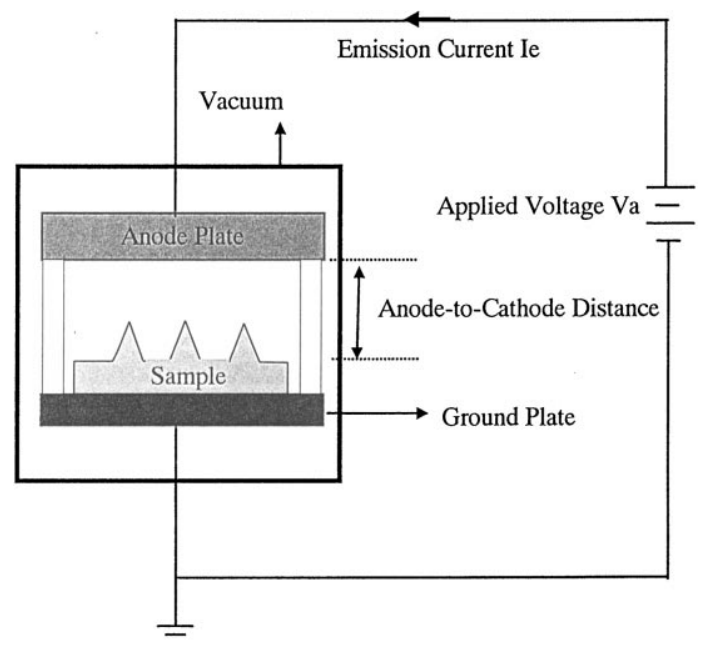

Fig. 3. Schematics of the set-up for field emission measurement. is a high-resolution field emission SEM, allowing a magnification of up to 300000 . The composition of the sample was analyzed by a Perkin Elmer scanning Auger nanoprobe system (SAN760), which allows depth profile data to be acquired from an area as small as $500 \AA$. Further composition and chemical bonding information were determined by X-ray photooelectron spectroscopy (XPS) using a Perkin Elmer Phi 1600 ESCA system. $\mathrm{MgK} \alpha$ radiation with an energy of $1253.6 \mathrm{eV}$ was used as the source with a linewidth of $0.7 \mathrm{eV}$. Depth profiling with the XPS was performed by $4 \mathrm{kV} \mathrm{Ar}$ ion-beam bombardment of the sample.

\section{Results and discussion}

\subsection{Emission from $B A C$ silicon tips}

Typical emission characteristics from the $50 \times 50 \mathrm{BAC}$ silicon tips are shown in Fig. 4. A turn-on field of $20 \mathrm{~V} / \mu \mathrm{m}$ and an emission current of $120 \mu \mathrm{A}$ at $36.7 \mathrm{~V} / \mu \mathrm{m}$ were achieved. The $\mathrm{F}-\mathrm{N}$ plot in the inset of Fig. 4 gave an effective barrier height of $0.017 \mathrm{eV}$, assuming a field enhancement factor of 31.6 from geometrical calculations. Note that the total current instead of current density is specified in this case due to the fact that it is an array of tips instead of a plain uniform film in our study. Therefore, it is meaningless to compare the current density as in the latter cases in this paper. In practical applications, the total current divided by the number of tips yielding the emission current per tip provides the most meaningful values.

\subsection{Emission from carbon nanotubes}

Typical emission characteristics of carbon nanotubes are shown in Fig. 5, in which a turn-on field strength of $10 \mathrm{~V} / \mu \mathrm{m}$ and a maximum current of $3.8 \mathrm{~mA}$ at $16.7 \mathrm{~V} / \mu \mathrm{m}$ were demonstrated. Multiple emission peaks were observed in Fig. 5, indicating emission site damage in the film and the subsequent appearance of newer emission sites at higher voltages, which is quite common in most carbon-nanotube samples. The phenomenon that several groups of emission sites coexist in a carbonnanotube sample can be attributed to the different height of the nanotube, various diameters, and the different curvature or different types of tubes. Further studies are needed to determine the role of each factor on the emission at different stages. The $\mathrm{F}-\mathrm{N}$ plot in the inset of Fig. 5 shows multiple emission groups, of which each individual group showed a linear relationship that follows the $\mathrm{F}-\mathrm{N}$ equation.

Taking into account a total number of 2500 squares in each array and a $64 \mu \mathrm{m}^{2}$ area for each square, one obtains a current density of $2.6 \mathrm{~A} / \mathrm{cm}^{2}$ at $16.7 \mathrm{~V} / \mathrm{cm}$, which is far beyond the emission current density of 


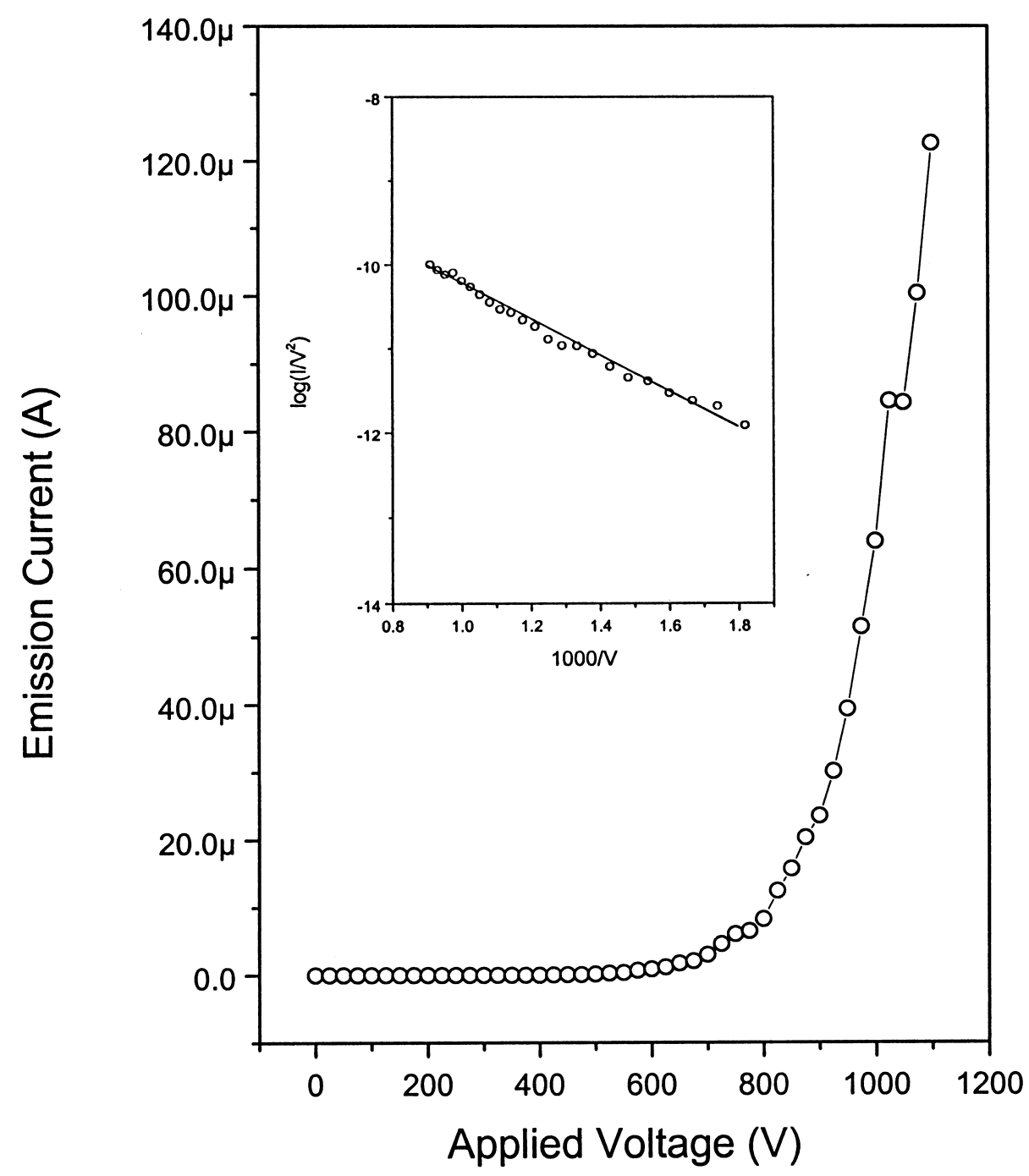

Fig. 4. Field emission $I-V$ curve and the corresponding F-N plot (inset) of $50 \times 50$ BAC silicon microtip arrays.

other materials at a comparable field. It still needs to be verified whether a larger-area carbon-nanotube sample will have the same performance as the arraytype sample. None the less, the arrayed carbon nanotubes demonstrate a great potential for various electron sources applications including that for microwave devices.

\subsection{Emission from SiCN films}

The electron emissions of three $\mathrm{SiCN}$ films are shown in Fig. 6, in which a turn-on field of $13 \mathrm{~V} / \mu \mathrm{m}$ and a maximum current density of $0.7 \mathrm{~mA} / \mathrm{cm}^{2}$ at $36.7 \mathrm{~V} / \mu \mathrm{m}$ have been demonstrated. The current density is relatively small, compared with that of carbon nanotubes. However, the $0.7 \mathrm{~mA} / \mathrm{cm}^{2}$ emission current density is several orders of magnitude higher than that of ordinary silicon or diamond films. The three sets of data from the top, middle, and bottom shown in Fig. 6 are taken from three samples of composition ratios [C]:[Si]:[N] of 22:21:57, 13:32:55, and 0:41:59, respectively. It is quite clear that carbon incorporation into the film is essential for the field emission enhancement. Meanwhile, the origins of signal oscillation that occurs at a high applied field are still unclear and will be investigated further. The F-N plots in Fig. 6 show different slopes for samples of various composition, which can be attributed to the different barrier heights of the films.

\subsection{Emission from SiCN nanorods}

Fig. 7 shows the emission characteristics of a SiCN nanorod sample. Compared to that of $\mathrm{SiCN}$ films, a higher emission current of $1.5 \mathrm{~mA} / \mathrm{cm}^{2}$ at $35 \mathrm{~V} / \mu \mathrm{m}$ with a turn-on field of $10 \mathrm{~V} / \mu \mathrm{m}$ was observed. The enhancement in emission properties can be attributed to the geometric factor of the nanorod structure as well as the change in barrier height due to the variation in 


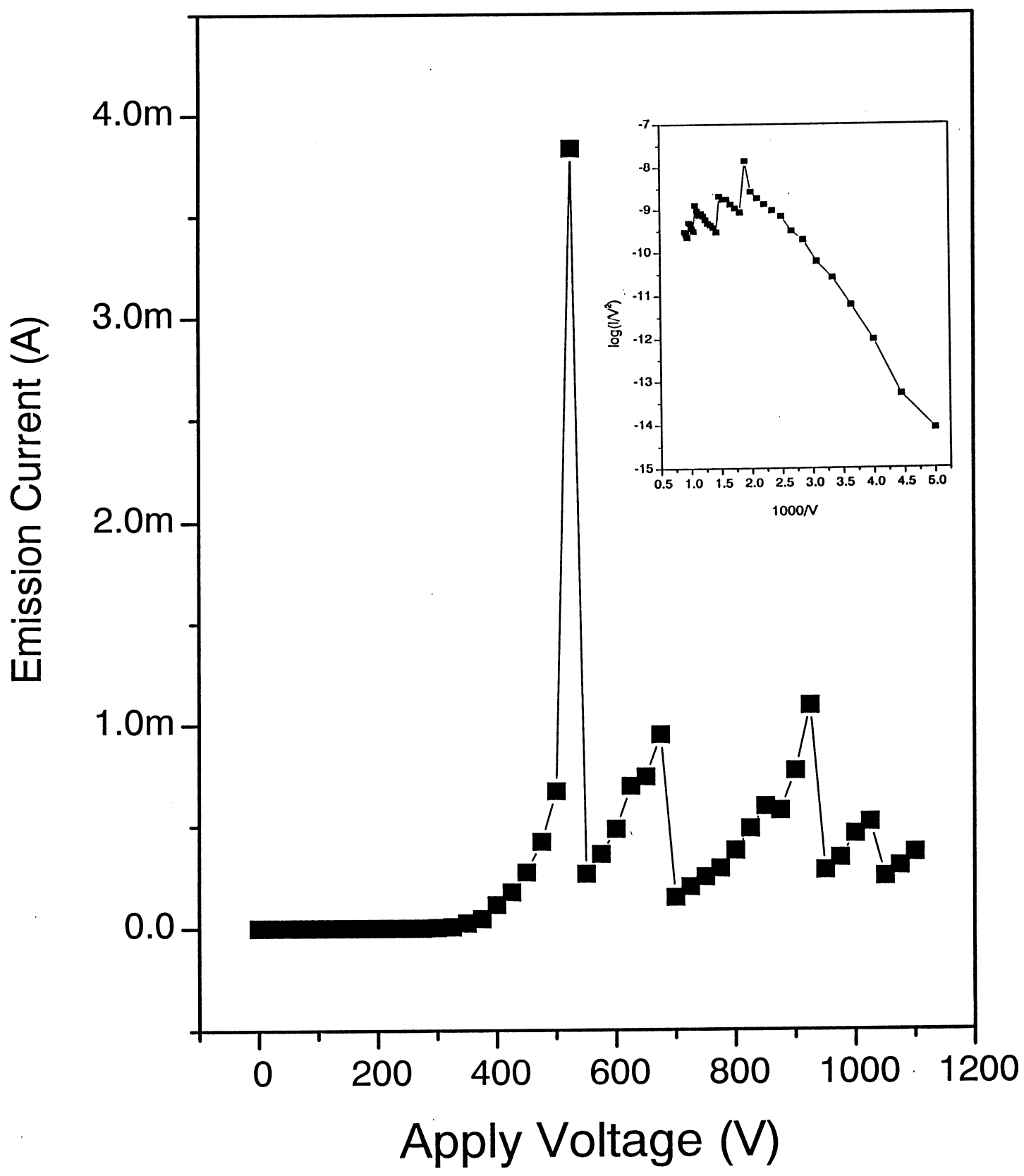

Fig. 5. Field emission $I-V$ curve and the corresponding $\mathrm{F}-\mathrm{N}$ plot (inset) of an array containing the $50 \times 50$ squares of carbon nanotubes.

composition/structure of the material. Remarkably, the F-N plot in the inset of Fig. 7 shows an excellent linear relationship over the entire range of applied field, indicating ideal field emission characteristics.

The Fowler-Nordheim description of the field emission can be expressed as

$J=A \frac{F^{2}}{\phi} \exp \left(\frac{-B \phi^{3 / 2}}{F}\right)$

where $A=0.014$ and $B=6.8 \times 10^{9}$ are constants. The units in the Fowler-Nordheim equation are: current density, $J\left(\mathrm{~A} / \mathrm{m}^{2}\right), F=\beta V / d$ with voltage, $V$ (volts), distance, $d(\mathrm{~m}), \phi$, the effective barrier height in $(\mathrm{eV})$, and $\beta$ the field enhancement factor that depends on the emitter geometry. Since there are only two parameters, $\beta$ and $\phi$, in this equation, a linear fit in the $\mathrm{F}-\mathrm{N}$ plot allows us to derive the field enhancement factor as well as the effective barrier height of the emitter, which can provide an insight into the physics of the field emission. However, the current density in the Fowler-Nordheim equation is often not well defined due to the ambiguity in the emission area, which is difficult to determine especially for materials of an irregular surface morphol- 


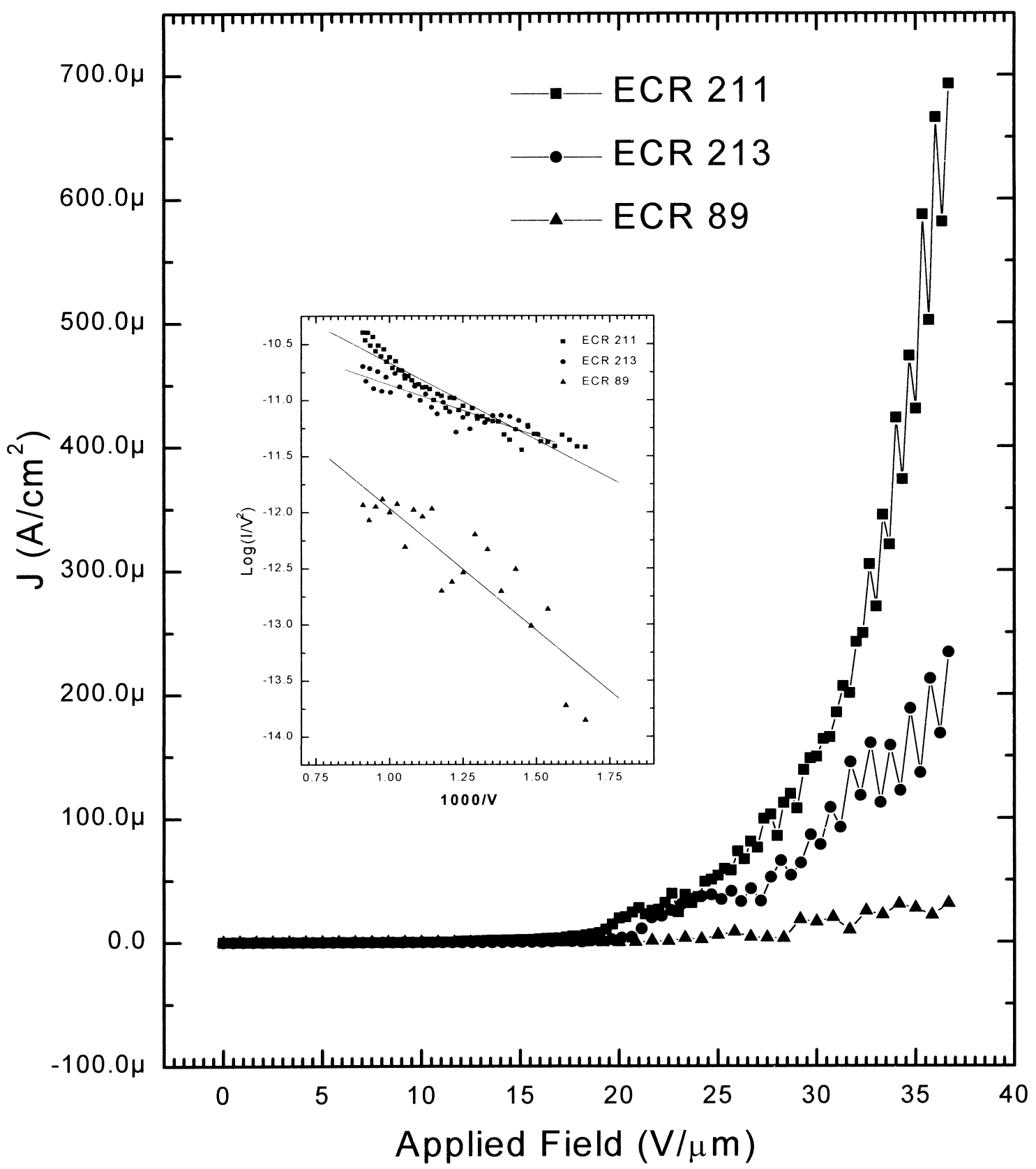

Fig. 6. Field emission $I-V$ curve and the corresponding F-N plot (inset) of three SiCN films with composition ratios [C]:[Si]:[N] of 22:21:57( $\mathbf{\square})$, 13:32:55(@), and 0:41:59( $\mathbf{\Delta})$.

ogy like nano-crystallites, nanotubes, and nanorods. Therefore, there are three unknown parameters, $\beta, \phi$, and the emission area, in the Fowler-Nordheim equation instead of two parameters as mentioned earlier. Apparently, a linear fit in $\mathrm{F}-\mathrm{N}$ plot only provides two parameters, slope and intersection, from which it is not possible to determine all the unknown parameters. Therefore, instead of providing the key parameters, $\beta$ and $\phi$, an $\mathrm{F}-\mathrm{N}$ plot can only be used to exemplify the field emission characteristics.

\subsection{Stability comparison of the carbon nanotubes and SiCN nanorods}

While achieving the highest emission current at the lowest applied field is the goal in most field emission studies, the long-term stability of the emission is also an essential issue to be addressed. A comparison of the emission stability of a carbon-nanotube sample and a $\mathrm{SiCN}$-nanorod sample is shown in Fig. 8. Although the carbon-nanotube sample showed a higher emission cur- 


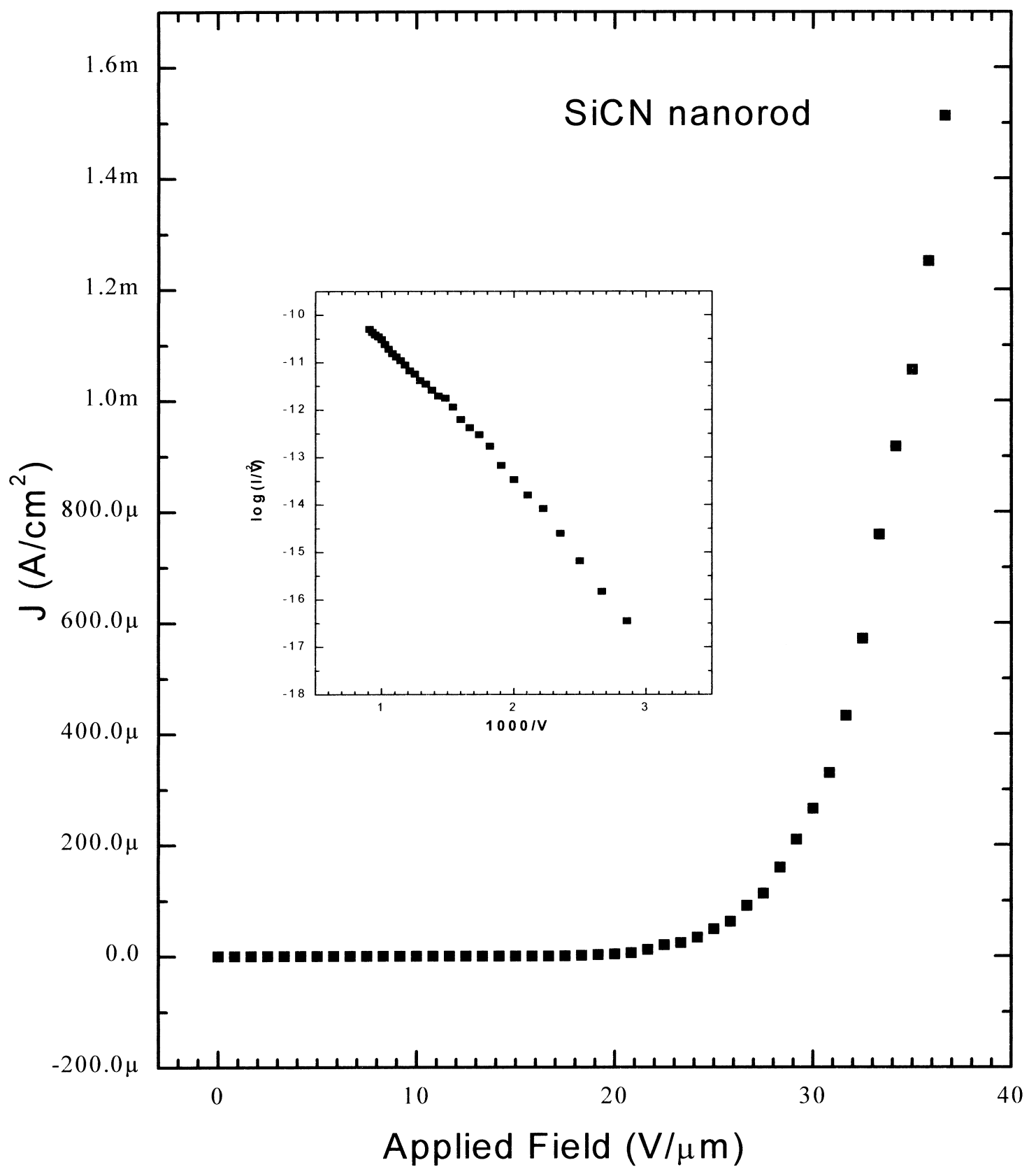

Fig. 7. Field emission $I-V$ curve and the corresponding $\mathrm{F}-\mathrm{N}$ plot (inset) of SiCN nanorods.

rent $(3.8 \mathrm{~mA}$ for an array of $50 \times 50$ squares), the emission current decayed to $15 \%$ of the initial value after $1000 \mathrm{~s}$ of operation. In contrast, the $\mathrm{SiCN}$ nanorods showed a lower emission current $\left(1.5 \mathrm{~mA} / \mathrm{cm}^{2}\right)$, but the decay was much slower, about $50 \%$ decay after $1000 \mathrm{~s}$ of operation. It should be pointed out that the stability measurement has not been standardized so far. In our case, an operating condition near the maximum emission current is chosen for the test. The decay characteristics will be very different if we choose another test condition such as half the emission current. Therefore, it would be wise to define the emission stability by the stressed emission at either specific current densities or at a specific applied field.

\section{Conclusion}

Among the four types of materials studied for electron field emission, carbon nanotubes deposited by MWPECVD demonstrated the highest emission current, which is several orders of magnitude higher than that 


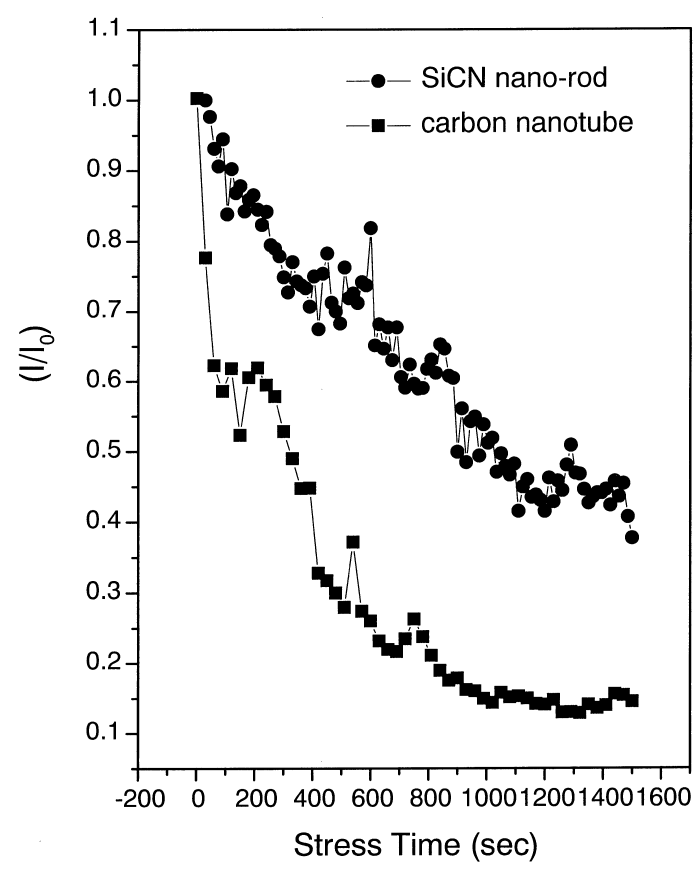

Fig. 8. Long-term stability of the field emission of SiCN nanorod ( and carbon nanotubes $(\mathbf{\square})$.

of diamond films. However, SiCN films and nanorods showed a moderate emission current, which is comparable to most of the 'good' emitters. However, the stability of the emitters should be emphasized to meet the requirement of practical applications. Moreover, the issue of deriving an effective barrier height and the field enhancement factor in Fowler-Nordhiem equation is addressed. It is concluded that a definitive effective emission surface area is needed to solve the $\mathrm{F}-\mathrm{N}$ equation completely and to obtain all the physical parameters.

\section{Acknowledgements}

Financial support for this project from National Science Council, Taiwan, under contract no. NSC-88-2113-M-001-021 and NSC-88-2112-M-002-022 is gratefully acknowledged. Two of the authors, Dr J.J. Wu and Dr P.D. Kichambare acknowledge the postdoctoral fellowship awarded by the National Science Council, Taiwan

\section{References}

[1] C. Wang, A. Garcia, D.C. Ingram, M.E. Kordesh, Electron. Lett. 27 (1991) 1459.

[2] M.W. Geis, N.N. Efremov, J.D. Woodhouse, M.D. McAleese, M. Marchywka, D.G. Socker, J.F. Hochedez, IEEE Electron Device Lett. 12 (1991) 456.

[3] M.W. Geis, J.C. Angus, Sci. Am. (1992) 84 October.

[4] W. Zhu, G.P. Kochanski, S. Jin, L. Seibles, J. Appl. Phys. 78 (1995) 2707.

[5] B.S. Satyanarayana, A. Hart, W.I. Milne, J. Robertson, Appl. Phys. Lett. 71 (1997) 1430.

[6] K. Okano, S. Koizumi, S. Ravi, P. Silva, G.A.J. Amaratunga, Nature 389 (1996) 140.

[7] L. Diederich, O.M. Kuttel, P. Aebi, L. Schlapbach, Diamond Relat. Mater. 8 (1999) 743.

[8] P.D. Kichambare, F.G. Tarntair, L.C. Chen, K.H. Chen, H.C. Cheng, J. Vac. Sci. Tech. (1999) (communication).

[9] L.C. Chen, T.R. Lu, C.T. Kuo, D.M. Bhusari, J.J. Wu, K.H. Chen, T.M. Chen, Appl. Phys. Lett. 72 (1998) 3449.

[10] L.C. Chen, C.Y. Yang, D.M. Bhusari, K.H. Chen, M.C. Lin, J.C. Lin, T.J. Chuang, Diamond Relat. Mater. 5 (1996) 514.

[11] K.H. Chen, J.J. Wu, S.L. Wei, C.Y. Wen, L.C. Chen, P.F. Kuo, Y.F. Chen, in: Handbook of Advance Electronic and Photonic Materials, H.S. Nalwa (Ed.). Academic Press, in press.

[12] T.W. Ebbesen, P.M. Ajayan, Nature 358 (1992) 220.

[13] K.H. Chen, J.J. Wu, C.Y. Wen, L.C. Chen, C.W. Fan, P.F. Kuo, Y.F. Chen, Thin Solid Films 355-356 (1999) 205. 